\title{
Quality Assessment of Volume Compression Approaches Using Isovalue Clustering
}

\author{
Bo Ma ${ }^{\mathrm{a}}$, Susanne K. Suter ${ }^{\mathrm{a}, \mathrm{b}}$, Alireza Entezari, Senior Member, IEEE. ${ }^{\mathrm{a}}$ \\ ${ }^{a}$ Department of Computer E Information Science E Engineering, University of Florida, Gainesville, FL, USA. \\ ${ }^{b}$ Anthropological Institute and Museum, University of Zurich, Zurich, Switzerland.
}

\begin{abstract}
Visualization of volumetric data has been widely used for exploration of data from scientific simulations and biomedical imaging. Despite advances of GPU-assisted rendering, which has become the state-of-art in direct volume rendering, still many volumetric data sets are too large to be visualized interactively. Therefore, compression-domain rendering approaches are used in visualization processes in order to reduce the amount of data sent to the GPU and thus speed up interactive visualization. Hence, reliable tools to assess the quality of the reconstructed 3D data are of great importance, influencing the effectiveness of the visualization. However, numerical error analysis approaches such as mean-squared-based metrics are often inconsistent with perceived visual quality. We propose a structural volume quality assessment approach for 3D scalar volume based on the human visual system (HVS). Our approach consists of two stages: First, we provide an interactive tool for extracting significant volume features via isosurfaces from a 3D scalar field using an isovalue classification process. Second, we propose a structural volume quality assessment (VQA) metric that employs representative isosurfaces as benchmark structures. For this purpose, we use a recently developed perceptual-based mesh quality metric [1] to assess the visual quality of compressed 3D scalar fields. Our experiments on a number of benchmark data sets suggest that, compared to existing methods, the proposed isovalue classification approach provides a more distinct set of isosurfaces that are more representative of the complexity of the data sets. We examine a number of widely used compression approaches, namely, discrete wavelet transform, discrete cosine transform, and tensor approximation, to establish the utility of our volume quality assessment approach.
\end{abstract}

Keywords: Volume quality assessment, volume rendering, isosurfaces, volume compression approaches, isovalue clustering.

\section{Introduction}

2 Volume visualization has become an indispensable tool in 3 volumetric data analysis and continues to facilitate discovery in 4 a broad range of applications from scientific computing (e.g., 5 simulations) to biomedical imaging (e.g., CT and MR). Di6 rect visualization algorithms make use of a user-defined trans7 fer function to assign color and opacity to the scalar field data 8 while indirect visualization requires the user to provide spe9 cific data values for extracting and rendering an isosurface. In 10 both paradigms, the user's input directly impacts the features 11 selected for visualization.

12 Direct Volume Rendering (DVR) [2] approaches allow sci${ }_{13}$ entists to visually inspect and explore a volume by simulating ${ }_{14}$ emission, absorption, and scattering of light as it travels through 15 the volume rather than explicitly extract geometric surfaces ${ }_{16}$ from the data. In visualization of large-scale data sets, DVR 17 approaches are often limited by the memory and computing re18 sources needed for rendering; hence, compression approaches 19 are commonly integrated to the visualization process [3].

20 Compression approaches use compact data representations, 21 which require fewer bits for encoding than what is needed for 22 the original volume. To generate a compact representation, a 23 routine decomposes a data set through a mathematical frame24 work or learned model into a set of bases and coefficients, which
25 are usually less error-prone to data reduction. Lossless algo${ }_{26}$ rithms are those that ensure maximum accuracy in the recon27 struction and guarantee zero error. Lossy algorithms produce an 28 approximation of the reconstructed volume up to a tolerated ap29 proximation error. Compact representations are often initially ${ }_{30}$ lossless and dense. However, higher compression ratios can be ${ }_{31}$ achieved by thresholding or encoding the coefficients. A recent ${ }_{32}$ example for an advanced encoding strategy has been proposed зз with fixed-rate compression of floating point coefficients [4]. ${ }_{34}$ Since data reduction becomes an essential step in large-scale ${ }_{35}$ data visualization, as recently shown with lossy compression of ${ }_{36}$ seismic tomography [5], reliable tools to assess the quality of 37 the reconstructed 3D data are of great importance. The quality ${ }_{38}$ assessment of the compressed data directly affects the chosen ${ }_{39}$ compression rates and influences the usability and effectiveness 40 of the visualization.

41 The volumetric approximation quality is either measured in 42 the reconstructed data set, i.e., in the data domain, or in the ${ }_{43}$ aggregated final 2D visualization, i.e., in the image domain. ${ }_{44}$ In case of volume visualization, the approximation quality is 45 usually measured numerically in the data domain in terms of 46 rate-distortion, which is a ratio between the achieved approxi${ }_{47}$ mation quality and the data reduction level. Frequently, the ap${ }_{48}$ proximation quality is measured using the mean-squared error ${ }_{49}$ (MSE) between the original and the approximation. However, 
50 the MSE measures a distortion rather than the perceived visual ${ }_{51}$ quality. The limitations of the MSE have been noted in image 52 processing [6], mesh processing [7], as well as for comparing ${ }_{53} 3 \mathrm{D}$ compression approaches [8].

54 One important characteristic of volume rendering is that only 55 subsets of the volumetric data, i.e., features of interest, are se56 lected and visualized in the final image [9]. A volumetric data 57 set contains a large number of isosurfaces at different isoval58 ues of the scalar field. In contrast, the volumetric structure is 59 only characterized by a finite number of representative isosur${ }_{60}$ faces that segment the data into important structures. The re${ }_{61}$ construction quality of these representative isosurfaces directly 62 portrays the visualization of compressed data. For a similar rea${ }_{63}$ son, Ljung et al. [10] proposed to evaluate the distortion of com${ }_{64}$ pressed data in the rendered images since the distortion of the 65 reconstructed (whole) volume has a reduced correspondence to ${ }_{66}$ the final image. That is, large subsets of the volume will give 67 little or no contribution to the rendering.

${ }_{68}$ In our approach, we propose to directly assess the quality 69 of the reconstructed volume in the data-domain. Instead of 70 conducting quality assessment on the whole volume, we lever71 age the characteristics of volume rendering and use representa72 tive isosurfaces as benchmark structures to evaluate the visual ${ }_{73}$ quality of compressed 3D scalar fields. Specifically, we pro${ }_{74}$ pose a two-stage approach to conduct volume quality assess75 ment (VQA). First, we cluster isovalues of a given data set and ${ }_{76}$ identify its representative isosurfaces (see Fig. 1(a)). Second, 77 we propose a structural VQA metric that examines the quality 78 of compressed volumetric data by evaluating its representative 79 isosurfaces using the tensor-based perceptual distance measure ${ }_{80}$ (TPDM) [1] that simulates human visual system (HVS) (see ${ }_{81}$ Fig. 1(b)).

82 The paper is organized as follows. In Section 3.1, we discuss 83 the choices of candidate isosurface similarity metrics for our ${ }_{84}$ proposed isovalue classification algorithm, presented in Sec${ }_{85}$ tion 3.2. In Section 3.3, a set of experiments analyzing our pro${ }_{86}$ posed approach on simulation and real data sets is conducted. ${ }_{87}$ Finally, in Section 4, we demonstrate a perceptual VQA metric 88 using representative isosurfaces to assess the visual quality of ${ }_{89}$ compressed 3D scalar fields.

\section{2. Related Work}

\section{${ }_{91}$ 2.1. Salient isovalues}

92 Bruckner and Möller [11] classified volumes by defining a ${ }_{93}$ similarity metric for the analysis of salient isovalues of a given ${ }_{94} 3 \mathrm{D}$ scalar field data set. The similarity between two specific iso95 surfaces is measured by the mutual information between their ${ }_{96}$ respective distance transforms (viewed as probability density 97 functions). Their isosurface similarity map (ISM) provides the 98 similarity information for any pair of isosurfaces present in a ${ }_{99}$ given data set. Based on the ISM, they apply a recursive pri100 ority queue algorithm to identify the most representative iso101 surface, i.e., the isosurface that is most similar to all other iso102 surfaces. The efficacy of the distance transform for accurately ${ }_{103}$ representing the geometry of an isosurface highly depends on
104 its sampled resolution and accurate reconstruction of the ge105 ometry from the distance transform is a challenging task [12]. 106 Moreover, the values of the distance transform that are relevant 107 to detailed surface geometry are only in the proximity of the 108 isosurface. Therefore, geometry-relevant information are not 109 well represented in a global measure (e.g., histogram) of the 110 distance transform.

\section{2.2. Compression-domain DVR}

12 Numerous approaches have been proposed in compression${ }_{113}$ domain DVR, as can be looked up in [3]. Examples of com114 pression approaches using pre-defined bases are the discrete ${ }_{115}$ Fourier transform (DFT) [13], the discrete cosine transform ${ }_{116}$ (DCT) [14], and the discrete wavelet transform (DWT) [15]. 117 The DFT decomposes the data into the frequency domain by 118 using sine and cosine waves as basis. DFT is known to be recep119 tive to periodic structures. DCT represents a simplified version 120 of the DFT as it uses only cosine waves and outputs only real 121 values. The DWT is decomposing the data with more complex 122 functions not only into the frequency domain, but also gives ac${ }_{123}$ cess to the spatial domain. Traditionally, the DWT is applied 124 in a multi-resolution fashion, what makes the DWT well-fitted 125 and a popular approach for multi-resolution visualization appli126 cations.

${ }_{127}$ Compression approaches using learned bases are dictionar${ }_{28}$ ies such as vector quantization (VQ) [16] or sparse coding 129 (SC) [17], and tensor approximation (TA) [18]. VQ learns dic130 tionary elements, i.e., small-sized volumes, that can be used ${ }_{131}$ to represent a scalar field through a lookup table. In SC, the ${ }_{132}$ general idea of VQ is further developed which uses linear com${ }_{133}$ binations of dictionary elements to represent a data set. TA is 134 basically a higher-order principal component analysis that sta135 tistically evaluates the major data components and represents ${ }_{136}$ them in vector bases that are mapped to a volume through co${ }_{137}$ efficients. The learned bases approaches learn a compact struc138 ture of the data set by minimize the energy within a data set such ${ }_{139}$ that as few coefficients as possible are needed. In our work, we 140 propose a structural VQA metric to compare those compression 141 approaches.

\section{2.3. Quality assessment of compressed data}

${ }_{143}$ MSE is a commonly used technique for numerical error anal144 ysis which is often limited by its inconsistency with perceived 145 visual quality. In image processing, the limitations of MSE146 like error analysis are addressed by designing metrics that sim147 ulate the HVS more closely. The HVS is sensitive to visual ${ }_{148}$ signals such as luminance, contrast, frequency content, or in149 teractions between different signal components, what is mod150 eled with the contrast sensitivity function (CFS). On this basis, 151 the Structural Similarity (SSIM) index [19], triggered numer152 ous image quality assessment (IQA) approaches and a review ${ }_{153}$ of the approaches based on SSIM is gathered in [20].

${ }_{154}$ The most recent IQA tools focus on contour-based ap155 proaches [21, 22, 23, 24], which are motivated by the fact that ${ }_{156}$ the HVS can detect the significant underlying features, even in 157 lower-quality images. These approaches can be of particular 


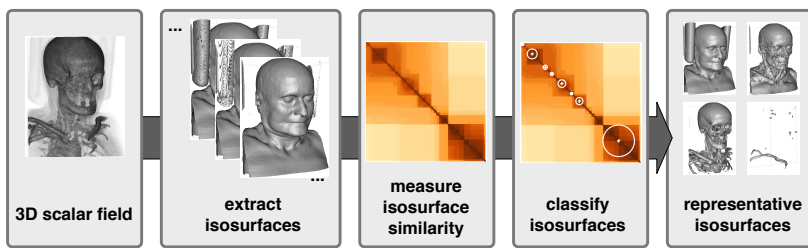

(a) Workflow of the proposed isovalue classification.
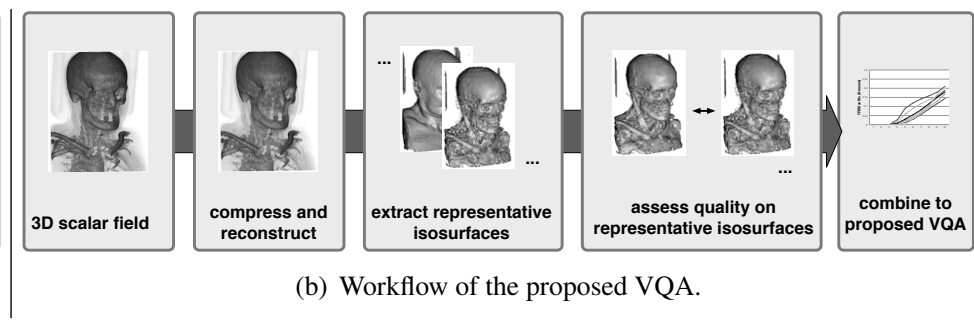

(b) Workflow of the proposed VQA

Figure 1

158 value when working with lossy data, where only approxima159 tions are available, but features that are relevant to the target 160 visualization need to be preserved. The advances in IQA stress ${ }_{161}$ the usefulness of tools that simulate the HVS in order to avoid ${ }_{162}$ cumbersome evaluation by a user/expert for every single image. ${ }_{163}$ To the best of our knowledge, structural VQA, analogous ${ }_{164}$ to IQA, have not yet been proposed for 3D scalar field data. 165 To fill this gap, we propose a VQA metric that uses signifi166 cant benchmark structures representing relevant data features ${ }_{167}$ to assess the visual quality of compressed 3D scalar fields. To 168 achieve this goal, we rely on algorithms that classify a 3D volu169 metric data set into relevant distinct structures, represented with 170 isosurfaces.

171 The closest work to VQA is the one on saliency maps [25], 172 a model to help users find salient and most relevant areas in 2D 173 or $3 \mathrm{D}$ visualizations. In contrast to our proposed work, their 174 output is a visual 2D map rather than an aggregated numeri$175 \mathrm{cal}$ value as tool output. Other methods treat data reductions 176 as sources of uncertainty, and quantifying the impact of uncer177 tainty has been studied in the context of isosurface visualiza178 tion [26, 27] as well as DVR [28].

\section{3. Isovalue Classification}

\section{3.1. Analysis of isosurface similarities}

181 An isosurface (level-set), $S_{\alpha}$, of a trivariate function $f$ is the 182 locus of all points $\mathbf{x}=(x, y, z)$ in space where $f$ attains the value ${ }_{183} \alpha$ :

$$
S_{\alpha}=\left\{\mathbf{x} \in \mathbb{R}^{3} \mid f(\mathbf{x})=\alpha\right\}
$$

184 Given samples of $f$ on a Cartesian grid, isosurface extraction 185 algorithms such as the Marching Cubes [29] produce a trian${ }_{186}$ gulation that approximates $S_{\alpha}$. The isovalues of interest for a 187 given data set depend on the range of $f$. They are usually a finite 188 set of isovalues selected depending on the quantization level of 189 the data. For instance, for byte valued data sets, the range of 190 isovalues is typically $\mathcal{I}=[0,255]$; however, non-integer iso191 values may be of interest for a particular data set. Often many 192 isosurfaces of a given data set are similar and exhibit the same 193 features; therefore, we are often only interested in a small sub194 set of all isovalues that represents the diversity of the features 195 present in the data set.

${ }_{196}$ In order to obtain a representative set of isovalues, we first 197 identify whether two given isovalues correspond to surfaces
198 (extracted using Marching Cubes algorithm) that represent sim199 ilar features from the underlying volumetric data. Such two 200 isovalues form a group of similar surfaces. On the other hand, 201 when the surfaces, corresponding to two isovalues, represent 202 different features of the data set they are to form different 203 groups.

204 We employ the notion of Hausdorff distance to discern iso205 surfaces of a data set and define similarities within a range of 206 isovalues. The Hausdorff distance has been used for shape 207 analysis (e.g., [30]). The key advantage of Hausdorff dis208 tance is that it allows us to express the difference between 209 any two arbitrary surfaces, connected or not. Spectral or 210 histogram-based approaches are difficult to employ for isosur211 faces since they are often composed of disconnected (open) sur212 faces [31]. Reference-to-distortion mesh quality metrics [7] re213 quire a minimum degree of shape correspondence between two 214 meshes. Some of those metrics assume the same mesh connec215 tivity, while others perform approximations to map two similar 216 meshes by sampling points between them. Therefore, the shape 217 deformation or reference-to-distortion metrics can not be used 218 to measure similarities between vastly different isosurfaces en${ }_{219}$ countered in 3D scalar field data.

Formally, we denote the distance between two isovalues $\alpha, \beta \in \mathcal{I}$ as the Hausdorff distance between their respective isosurfaces:

$$
i \operatorname{sodist}(\alpha, \beta)=d_{H}\left(S_{\alpha}, S_{\beta}\right) .
$$

220 Here $d_{H}\left(S, S^{\prime}\right)=\max \left(d\left(S, S^{\prime}\right), d\left(S^{\prime}, S\right)\right)$ refers to the 221 symmetric mean Hausdorff distance where $d\left(S, S^{\prime}\right)=$ ${ }_{222} \sup _{\mathbf{x} \in S} \inf {\mathbf{y} \in S^{\prime}}\|\mathbf{x}-\mathbf{y}\|$. The classical Hausdorff distance is the 223 maximum of the smallest distances between two isosurfaces ${ }_{224} S_{\alpha}$ and $S_{\beta}$, which is an asymmetric distance. Depending on 225 the geometry of the two surfaces this can lead to situations 226 where $i \operatorname{sodist}(\alpha, \beta) \gg i \operatorname{sodist}(\beta, \alpha)$ or vice versa. A small one227 directional Hausdorff distance does not necessarily describe 228 surfaces that represent the same features in the volumetric data. ${ }_{229}$ Therefore, we use the symmetric Hausdorff distance as it is 230 commonly used in shape analysis [30]. Since the maximum ${ }_{231}$ Hausdorff distance is sensitive to outliers in one surface, we 232 also use the mean Hausdorff distance, which is the arithmetic 233 mean of all shortest distances between two surfaces.

${ }_{234}$ The similarity map for a range of isovalues $\mathcal{I}$ can be pre235 computed for any pair of isovalues in $\mathcal{I}$. Accordingly, this map ${ }_{236}$ depicts the relationship between any two surfaces present in the ${ }_{237}$ volumetric data. In the remainder of this paper, we refer to the 238 map based on the Hausdorff distance as HDM and to the map 
239 based on mutual information of the distance transform [11] as 240 ISM.

\section{3.2. Classification of isovalues}

${ }_{242}$ Considering a set of isovalues $\mathcal{I}$, the goal is to select a small ${ }_{243}$ subset, $\mathcal{K} \subset \mathcal{I}$, that represents the diversity of shapes in the ${ }_{244}$ surfaces corresponding to $\mathcal{I}$. The set of selected isovalues $\mathcal{K}$ 245 can be used for the exploration of features in the data set or de246 signing transfer functions for volume rendering. We can view 247 this as a quantization problem where the range of isovalues $\mathcal{I}$ 248 is quantized to a small set $\mathcal{K}$. Unlike in traditional quanti249 zation, the data points (isovalues) do not have a trivial vector 250 space embedding (e.g., sum of two isovalues represents a sur251 face that is possibly unrelated to the two isosurfaces). However, 252 we can consider the quantization problem as the discretization ${ }_{253}$ of the space of isovalues equipped with the distance as defined 254 in Eq. (2). This analogy allows us to employ Centroidal Voronoi ${ }_{255}$ Tessellation (CVT) to partition the isovalue space, an approach 256 that is widely used in quantization and clustering. In particu257 lar, we can employ Lloyd's algorithm for K-means clustering 258 in forming partitions of the isovalue space.

259 The search for the optimal set of isovalues $\mathcal{K}$ is an iterative 260 process similar to the Lloyd's algorithm; however, the cluster ${ }_{261}$ membership and centroids are determined based on the HDM. ${ }_{262}$ Moreover, we may use other notions of distance such as the ${ }_{263}$ ISM [11] for this process. We initialize $\mathcal{K}$ to a set of isoval${ }_{264}$ ues $\mathcal{K}:=\left\{\mu_{1}, \ldots, \mu_{\mathrm{K}}\right\}$, with $\mathrm{K}=|\mathcal{K}|$ representing the number of 265 desired representative isovalues. The choice of initial isovalues ${ }_{266}$ can be uniform throughout $\mathcal{I}$ or guided by isosurface area his267 tograms. The iterations for finding the optimal $\mathcal{K}$ are composed 268 of these two update steps:

- Isovalue Membership Update: For each isovalue $\alpha \in \mathcal{I}$ find the closest centroid isovalue:

$$
\mu=\underset{\mu \in \mathcal{K}}{\arg \min } i \operatorname{sodist}(\alpha, \mu) .
$$

Assign $\alpha$ to $C_{\mu}$ - the cluster represented by $\mu$.

- Centroid Isovalue Update: For each centroid, $\mu \in \mathcal{K}$, collect all isovalues $\alpha \in \mathcal{I}$ that have been assigned to $\mu$ to form the cluster $C_{\mu}$. Update the centroid $\mu$ by the isovalue whose isosurface is as close as possible to the rest of the cluster members.

$$
\mu=\underset{\alpha \in C_{\mu}}{\arg \min } \sum_{\beta \in C_{\mu}} i \operatorname{sodist}(\alpha, \beta)
$$

270 As every update step reduces the Hausdorff distances be271 tween the surfaces corresponding to isovalues in a cluster, the 272 iterations are guaranteed to converge to a minimum cluster as273 signment configuration. Similar to Lloyd's algorithm for K274 means clustering (and in CVT), the convergence to a global 275 minimum is more difficult to guarantee and requires a number 276 of random initializations [32, 33].

\section{3.3. Evaluation}

278 In [34], we have shown that the Hausdorff metric-based K279 means clustering outperformed the previous approach [11] in 280 terms of identifying representative isovalues. In this section, ${ }_{281}$ we briefly review the evaluation experiment.

We conducted a detailed analysis on an exemplar head CTA 283 dataset by varying (1) the classification approach, and (2) the 284 isosurface similarity metric. In order to evaluate the results, 285 we manually analyzed all the isosurfaces $S_{\alpha}$ extracted at the 286 isovalues $\mathcal{I}=[1,255]$ and defined feature groups that contain ${ }_{287}$ distinctive features of the CT head data set.

288 The manual visual feature inspection was performed by two 289 observers and resulted in seven feature groups as illustrated in 290 Fig. 2: isovalues 1-10 depict the patient head including clothes, 291 side props and a bandage, isovalues 11-29 show the patient 292 head with the side props only; isovalues 30-55 represent the 293 skin and only partial side props; isovalues 56-68 correspond 294 to soft tissues mostly below the skin; isovalues 69-101 repre295 sent the skeleton and vessels; isovalues 102-149 correspond to 296 partial bones and vessels; and isovalues 150-255 reveal details 297 such as two teeth, the eyeballs, and the prominent vessels with 298 contrast liquid. The group 102-149 covers a wide range of iso299 surfaces that represent varying degrees of partial bone/vessel 300 tissue. For visualization purposes, this group does not reveal 301 any semantic meaning. The group 150-255 includes almost 302 empty isosurfaces, which depict scarce representations of the ${ }_{303}$ detected features in that group.

We performed classification experiments using (a) the (max305 imum) HDM, (b) the mean HDM (mHDM), and (c) the in306 verse ISM (iISM) as underlying distance metric. The classi307 fication was run with $K=13$ clusters and the initial centroids ${ }_{308} \mathcal{K}:=\{10,30,50, \cdots, 230,250\}$. For the studied data set, this 309 number was picked such that we have enough clusters for representing each feature group. Concordant classification exper311 iments were conducted to find the first $K$ most representative 312 isosurfaces, as described in [11].

(3) ${ }_{313}$ All classification experiments resulted in some repeated rep${ }_{314}$ resentatives found for certain feature groups. In general, the 315 classification from [11] outputs more repeated representatives 316 than the ones from K-means clustering. In conclusion, the rep317 resentatives from the proposed isovalue classification using the $318 \mathrm{HDM}$ are closest to the manual inspection with respect to the 319 maximum number of distinct features. We refer readers to [34] 320 for details of the experiments.

\section{3.4. Discussion of clustering approaches}

322 We performed an extensive exploration of varying clustering ${ }_{323}$ approaches before we decided to use the simple K-means ap${ }_{324}$ proach. In fact, in unsupervised learning applications, K-means 325 is still often the tool of choice as it is simple to implement, de326 terministic, scales well for large amounts of data, and converges ${ }_{327}$ quickly. However, for K-means, the ground truth number of $\mathrm{K}$ 328 clusters needs to be known a priori what is often difficult or an 329 unknown. We focused on clustering approaches that estimate ${ }_{330}$ the number of $\mathrm{K}$ clusters.

The first concept that picked up that idea of estimating the ${ }_{332}$ number of $\mathrm{K}$ clusters is attributed to Pelleg \& Moore [35] who 


\begin{tabular}{|l|c|c|c|c|c|c}
\hline $\begin{array}{c}\text { group } \\
1-10\end{array}$ & $\begin{array}{c}\text { group } \\
11-29\end{array}$ & $\begin{array}{c}\text { group } \\
30-55\end{array}$ & $\begin{array}{c}\text { group } \\
56-68\end{array}$ & $\begin{array}{c}\text { group } \\
69-101\end{array}$ & $\begin{array}{c}\text { group } \\
102-149\end{array}$ & $\begin{array}{c}\text { group } \\
150-254\end{array}$ \\
\hline $\begin{array}{l}\text { head } \\
\text { clothes } \\
\text { props } \\
\text { bandage }\end{array}$ & $\begin{array}{l}\text { head } \\
\text { props }\end{array}$ & skin & $\begin{array}{l}\text { soft } \\
\text { tissues }\end{array}$ & $\begin{array}{l}\text { skeleton } \\
\text { vessels }\end{array}$ & $\begin{array}{l}\text { partial } \\
\text { bones } \\
\text { vessels }\end{array}$ & $\begin{array}{l}\text { details: } \\
\text { teeth } \\
\text { eyeballs } \\
\text { vessels }\end{array}$ \\
\hline \\
\hline
\end{tabular}

Figure 2: Feature groups of the CT head data set obtained by manual inspection. The first row depicts the isovalue ranges for each group. The second row contains the description of each feature group. The third row displays the exemplar isosurfaces from each feature group.

${ }_{333}$ proposed the X-means algorithm. X-means searches for the 334 most likely number of $\mathrm{X}$ clusters in an iterative splitting process 335 around the standard K-means algorithm. After every K-means 336 run, X-means measures the score of each (parent) cluster and ${ }_{337}$ the score of the two child clusters running $\mathrm{K}$-means with $\mathrm{K}=2$ $3 з 8$ on the subset corresponding to the parent cluster. Dependent on ззя the score value, X-means continues either with the parent or the 340 two child clusters.

${ }_{341}$ When using X-means for the proposed isovalue classifica342 tion, we most of the times did not arrive at a splitting of the ${ }_{343}$ clusters. Often, one of the children had a particularly bad like344 lihood score that prevented the parent cluster from splitting. We 345 explored X-means using likelihood score estimators such as the ${ }_{346}$ Bayesian Information Criterion (BIC) [35] and the Minimum 347 Noiseless Description Length (MNDL) scores [36], which both 348 rely on Gaussian distributed data. We assume that X-means 349 achieved poor results on our data, because we cannot assume a ${ }_{350}$ Gaussian distribution of the isovalue similarity.

351 The issue of finding the number of clusters in an automated 352 process for non-Gaussian distributed data was, for example, ad${ }_{353}$ dressed with the DP-means clustering approach proposed by ${ }_{354}$ Kulis \& Jordan [37]. DP-means is similar to K-means in its for355 mulation, but it is derived from a Dirichlet process (DP) mixture 356 model.

${ }_{357}$ We explored using DP-means, e.g., for the CT head data 358 set where we received a set of centroid isosurfaces $\mathcal{K}:=$ $359\{4,24,52,73,87,108,119,141,144,208\}$ on the HDM with ${ }_{360} \lambda=0.1942$. A number of centroid isovalues $\mathcal{K}:=$ ${ }_{361}\{4,24,52,87,108,208\}$ are overlapping with the results of $\mathrm{K}$ 362 means. However, the interesting feature group from isovalues ${ }_{363} 56-68$ is missing. Similar results were obtained with other data 364 sets. Since DP-means is dependent on the processing order of 365 the points, we further explored varying processing orders such 366 as increasing/decreasing isovalue order, isosurface area-based 367 processing order, or alternating processing orders.

368 Since the results did not significantly improve the clustering 369 results, we decided to use the most simple clustering approach, 370 i.e., K-means, and let the user explore the number of $\mathrm{K}$ clusters 371 in an interactive process with a graphical tool. In fact, using 372 DP-means means that the clustering depends on the distance pa${ }_{373}$ rameter $\lambda$, which is responsible for when to open new clusters, 374 which again requires an explorative process with user interac-

\begin{tabular}{rcr}
\hline Dataset & \multicolumn{2}{c}{ K-means clustering } \\
\cline { 2 - 3 } & \# Iterations & Time \\
\hline CT head & & \\
$(128 \times 128 \times 115)$ & 3 & $0.16 \mathrm{~s}$ \\
Fuel & & \\
$(64 \times 64 \times 64)$ & 3 & $0.14 \mathrm{~s}$ \\
Hydrogen & & \\
$(128 \times 128 \times 128)$ & 5 & $0.11 \mathrm{~s}$ \\
\hline
\end{tabular}

Table 1: The performance of K-means on the HDMs $(255 \times 255)$ of the exemplar data sets. System configuration: Intel Core i7 $3.6 \mathrm{GHz}$ CPU, 16 GB RAM.

375 tion. Besides, for a naive user, choosing $\lambda$ is not necessarily 376 more intuitive than choosing $\mathrm{K}$.

Finally, we performed a robustness check of K-means cen378 troids when varying K. Fig. 3 (a) shows the resulting centroids 379 from K-means runs for $\mathcal{K}=\{7,8, \cdots, 12\}$ on the three isosur380 face similarity metrics. The darker a bar, the more hits were 381 found for a centroid isovalue. Analyzing Fig. 3 (a), we con382 cluded that K-means gives most robust results on the HDM ${ }_{383}$ meaning that many centroids were chosen independent from ${ }_{384} \mathrm{~K}$. Similar to the results from the previous section, the feature ${ }_{385}$ group 150-255 is least robust with respect to a single centroid. ${ }_{386}$ We explain this with the same argument as previously that this 387 feature group has no clear semantic distinction of shapes. Fur388 thermore, as the number of clusters ranges from 7-12, some 389 new clusters are only formed with higher Ks. As the feature 390 group 150-255 comprises many isovalues, this range is more 391 likely to give birth to new centroids.

392 Fig. 3 (b) shows the centroid distribution for DP-means with 393 varying $\lambda=\{0.202,0.206,0.212, \cdots, 0.298\}$ resulting in $7-12$ 394 clusters. Even though the feature group 150-255 has more ro395 bust results with DP-means compared to K-means, it is less ro396 bust in the other visually more important feature groups. There397 fore, the centroid robustness check for DP-means leads to the 398 same conclusion as before, namely, that DP-means does not 399 give enough advantages in order to choose it instead of $\mathrm{K}$ 400 means. Therefore, we use in our continuing experiments K401 means on the HDM as isovalue classification approach.

\section{3.5. Performance}

${ }_{403}$ In order to identify representative isovalues, we developed a 404 tool where users can interactively explore the data by starting 405 with a relatively large $\mathrm{K}$ to find the expected number of distinct 406 features and then eliminate similar shapes found by K-means. 407 Thus, the performance of K-means for isovalue classification 408 determines the efficiency of such interactive exploration pro409 cess. Table 1 lists the performance of K-means clustering on 410 the HDMs of the exemplar data sets. The clustering works ef411 ficiently and K-means converges quickly, typically within 3-5 412 iterations as shown in the experiments. Note that the resolution ${ }_{413}$ of the data does not directly influence the performance of K- 


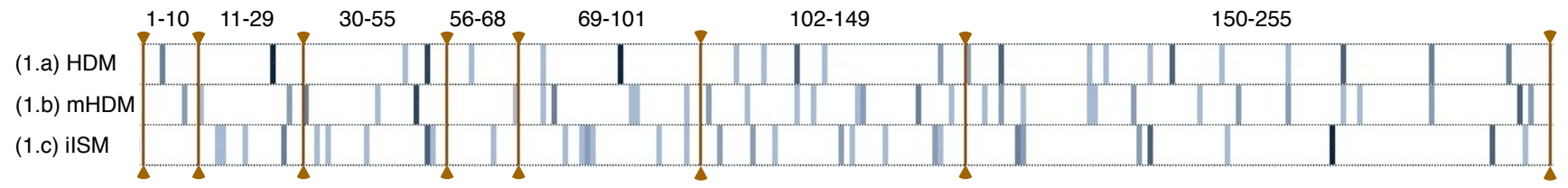

(a) Distribution of K-means centroid isovalues on HDM, mHDM and iISM for $K=7,8, \cdots, 12$.

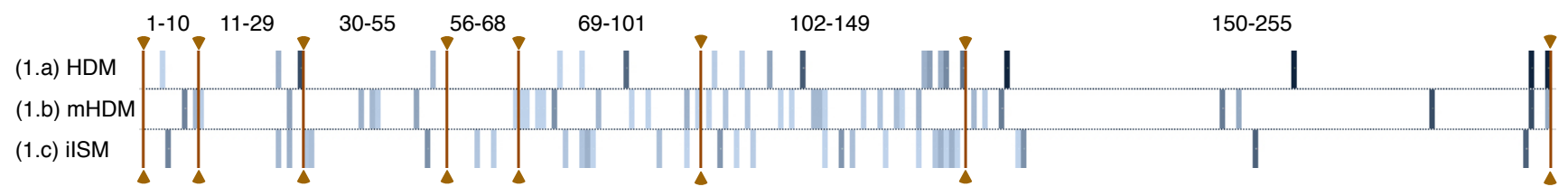

(b) Distribution of DP-means centroid isovalues on HDM, mHDM and iISM using a regular lambda increment.

Figure 3: Robustness check for K-means and DP-means. The centroid isovalues are represented by the vertical bars. The darker a bar, the more hits were found for a centroid isovalue.

${ }_{414}$ means clustering; however, it will increase the pre-computation 415 time of the similarity maps.

\section{${ }_{416}$ 4. Volume Quality Assessment}

417 Our motivation to design a new VQA originates from ${ }_{418}$ challenging problems that arise in comparing compression 419 approaches applied to 3D scalar fields, as known from 420 compression-domain DVR. In this section, we demonstrate how 421 we connect structural quality assessment to the introduced iso422 value classification.

\section{${ }_{423}$ 4.1. Motivation of the proposed VQA approach}

424 Despite the known significant shortcomings in identifying vi425 sually relevant information [6], numerical error analysis tools 426 such as MSE or SNR are still widely used for visual quality as${ }_{427}$ sessment in 3D scalar fields. In the image processing commu428 nity, the concept of structural similarity index (e.g., SSIM [19]) 429 has led to the development of numerous approaches that as430 sess the data quality using structural information that are rele${ }_{431}$ vant to the HVS, which in return leads to quality measures that ${ }_{432}$ correlate more closely with the human visual perception. A ${ }_{433}$ good overview of HVS inspired perceptual quality assessment 434 approaches can be found in [20]. Analogous structural informa435 tion approaches have been applied to mesh quality assessment 436 (MQA), summarized in [7]. To the best of our knowledge, how${ }_{437}$ ever, similar structual VQA approaches have not yet been pro438 posed.

439 Inspired by structural IQA and MQA, we propose to assess 440 the visual quality of $3 \mathrm{D}$ scalar fields based on a limited set ${ }_{441}$ of significant benchmark structures. Our work is motivated by ${ }_{442}$ Pinto et al. [22] who measure the contour degradation as ba${ }_{443}$ sis for an IQA measure. This idea is based on the sensitivity ${ }_{444}$ of the HVS to edges, which makes it effective on identifying 445 contours (or high contrasts) to recognize structures. In fact, ${ }_{446}$ the HVS is able to find contours and structures even on low${ }_{447}$ quality images. We propose to selectively measure the volume
448 quality of 3D scalar fields at significant 3D benchmark isosur${ }_{449}$ faces. In particular, we suggest to select the benchmark con450 tours such that they represent a wide range of distinct shapes ${ }_{451}$ contained in a 3D scalar field. Connecting this idea to the intro452 duced isovalue classification in Sec. 3.2, we propose to select 453 the benchmark contours specifically at the representative iso${ }_{454}$ surfaces (RIs), which each represent a set of distinct features in 455 the data.

456 In order to measure the degradation of an isosurface when 457 compression is applied, we use structural MQA. The basic idea 458 behind MQA is to define visual quality metrics that reflect the ${ }_{459}$ subjective judgement of the HVS. One approach, the Mesh ${ }_{460}$ Structural Distortion Measure (MSDM) [38] can be seen as an ${ }_{461}$ extension of the SSIM to 3D meshes. Basically, the MSDM 462 assesses differences in curvature statistics (amplitudes) com463 puted through a local moving window over the two meshes. ${ }_{464}$ The MSDM has one limitation, namely that the two meshes 465 under comparison need to consist of the same mesh topology, 466 i.e., the same number of vertices and connectivity. However, 467 meshes often underly transformations such as compression that 468 produce meshes of different topology. Two recently developed ${ }_{469}$ MQAs address this bottleneck. The Multiscale Mesh Structural 470 Distortion Measure (MSDM2) [39] performs a fast asymmetric 471 matching between the vertices of the two meshes. Moreover, ${ }_{472}$ Gaussian-weighted curvature statistics are computed at multi${ }_{473}$ ple scales to produce a global distortion map. The Tensor-based ${ }_{474}$ Perceptual Distance Measure (TPDM) [1] has taken the idea of ${ }_{475}$ MSDM2 one step further by incorporating further structural in476 formation, namely, the surface principal directions, defined by 477 the orthogonal directions of the minimum and maximum cur478 vatures. Additionally, the TPDM simulates the visual masking 479 effect through a roughness-based weighting of local distances. 480 Finally, the TPDM score is obtained through Minkowski pool481 ing of the weighted local tensor distances throughout the mesh 482 surface.

${ }_{483}$ For the VQA, we compute the degradation of the benchmark 484 contours using the most recently developed TPDM, which of${ }_{485}$ fers a good correspondence to the HVS verified with subjec- 
${ }_{486}$ tive databases. As we are extracting meshes on compressed ${ }_{487}$ 3D scalar field data, we rarely have meshes with the same con488 nectivity and hence rely on a MQA metric that uses a vertex 489 projection step for the analysis of the visual quality.

\section{4.2. Definition of the proposed VQA}

491 The basic workflow of the definition of the proposed VQA 492 for 3D scalar fields is illustrated in Fig. 1(b). That is, we com${ }_{493}$ press a $3 \mathrm{D}$ scalar field $\mathcal{A} \in \mathbb{R}^{x, y, z}$ and reconstruct it to its ap${ }_{494}$ proximation $\widetilde{\mathcal{A}} \in \mathbb{R}^{x, y, z}$. The approximation quality of $\widetilde{\mathcal{A}} \mathrm{de}-$ 495 pends on the chosen compression approach as well as on the 496 chosen compression ratio. In order to define the visual qual${ }_{497}$ ity of $\widetilde{\mathcal{A}}$, we extract from the reference $\mathcal{A}$ and the distortion ${ }_{498} \mathcal{\mathcal { A }}$ a set of $K$ benchmark isosurfaces $S$ at the learned represen499 tative isovalues $\mathcal{K}:=\left\{\mu_{1}, \ldots, \mu_{K}\right\}$, with $K=|\mathcal{K}|$, as obtained 500 from Sec. 3.2. For every corresponding isosurface pair $S_{\mu_{k}}$ and ${ }_{501} \widetilde{S}_{\mu_{k}}$, we individually assess the mesh quality $M Q_{T P D M}(S, \widetilde{S})$ as 502 computed with the TPDM. For the final VQA, we aggregate the ${ }_{503} M Q_{T P D M}(S, \widetilde{S})$ for every isosurface pair using the arithmetic 504 mean to receive one single volume quality score:

$$
V Q(\mathcal{A}, \widetilde{\mathcal{A}})=\frac{1}{K} \sum_{\mu_{k} \in \mathcal{K}} 1-M Q_{T P D M}\left(S_{\mu_{k}}, \widetilde{S}_{\mu_{k}}\right)
$$

505 The scores of the TPDM are normalized between $[0,1]$, 506 where the lower the score, the better the quality. Since the SSIM 507 and the SNR use a quality assessment of the higher the score 508 the better the visual quality, we inverted the TPDM scores in ${ }_{509}$ our aggregation to the $V Q(\mathcal{A}, \widetilde{\mathcal{A}})$ to make the comparison be${ }_{510}$ tween different VQA approaches more intuitive. Hence, the ${ }_{511} V Q(\mathcal{A}, \widetilde{\mathcal{A}})$ scores show a higher quality the closer to 1 .

${ }_{512}$ To further connect the proposed $V Q$ score to the perceptual 513 visual quality of compressed data, we employ a psychometric ${ }_{514}$ function, which plots subjective visual sensations against $V Q$ 515 scores. The subjective responses to various degrees of com516 pression are obtained from four observers. Each observer is 517 exposed to a set of RIs at various compression levels. At each 518 time, the subject is asked to compare the rendered approxima519 tion with the ground truth and assign a five-scale quality score 520 to the underlying approximation. The subjective quality score ${ }_{521}$ for the compressed volume is obtained from the mean quality 522 scores of its RIs from all observers. Then, we conduct a psycho${ }_{523}$ metric fitting between the $V Q$ scores and the subjective scores 524 using the cumulative Gaussian psychometric function [1]:

$$
p=\frac{1}{\sigma \sqrt{2 \pi}} \int_{-\infty}^{x} e^{-\frac{(t-\mu)^{2}}{2 \sigma^{2}}} d t
$$

${ }_{525}$ where $x$ is the raw $V Q$ score. The parameters $\mu=0.132$ and ${ }_{526} \sigma=0.034$ are obtained through a non-linear least squares fit${ }_{527}$ ting using the $V Q$ scores and subjective scores of the CT head ${ }_{528}$ data set. The psychometric function maps each $V Q$ score to a ${ }_{529}$ probability of visual quality between 0 (certainty of low visual ${ }_{530}$ quality) and 1 (certainty of high visual quality), which is easier ${ }_{531}$ to be understood by users. As shown in Fig. 4, the same psycho${ }_{532}$ metric function is used for other experimented data sets, where ${ }_{533}$ the plots show a good generalization of the fitted psychometric 534 function.

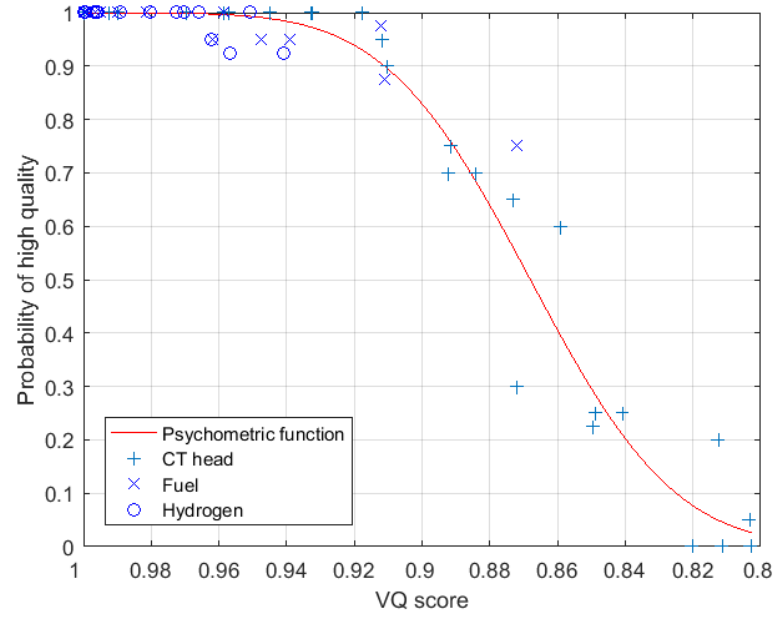

Figure 4: Psychometric function for the proposed quality metric plotted with $V Q$ score and $p$ pairs of all the experimented data sets with the compressions at level $100 \%, 75 \%, 54 \%, 37 \%, 25 \%, 15 \%, 8 \%, 4 \%, 1.5 \%$, and $0.5 \%$.

\section{4.3. Experiments with compression-domain VQA}

For the purpose of analyzing the proposed VQA, we mea${ }_{537}$ sured the reconstruction quality of three compressed exemplar ${ }_{538}$ 3D scalar fields. For approaches to compression we have cho539 sen DCT as a representative for frequency-domain transform, ${ }_{540}$ DWT as a representative for state-of-the-art multi-resolution ${ }_{541}$ decomposition, and TA as an example of a recent approach us542 ing a learned model. The initial decomposition of the 3D scalar 543 field was performed as a lossless process. The data reduction is 544 then applied on the decomposed/learned models. To be precise, 545 besides the thresholding of coefficients, we did not apply any ${ }_{546}$ further data compression approaches such as run-length encod547 ing or floating point quantization. The coefficient thresholding 548 for DCT and DWT was performed such that the least signifi549 cant coefficients were removed. For TA, the data reduction was 550 performed with Tucker tensor rank truncation [40].

For the comparison, we matched the number of coefficients ${ }_{552}$ (NCs) for DCT, DWT and TA. We purely calculated the NCs, 553 i.e., the DCT coefficients, the DWT coefficients and the core 554 tensor coefficients for TA. Elements of bases such as the 555 wavelet or the tensor bases do not contribute to our reported ${ }_{556} \mathrm{NCs}$. Our chosen data reduction levels are approximately at $557100 \%, 75 \%, 54 \%, 37 \%, 25 \%, 15 \%, 8 \%, 4 \%, 1.5 \%$, and $0.5 \%$ 558 of NCs compared to the original number of scalar field values, 559 which correspond to the NCS of a systematic tensor rank trun${ }_{560}$ cation. For the VQA, we reconstructed the 3D scalar fields for ${ }_{561}$ DCT, DWT and TA at every data reduction level. The resulting ${ }_{562}$ VQAs were analyzed with respect to (a) the visual degradation ${ }_{563}$ of benchmark contours, and (b) the measured visual quality. ${ }_{564}$ The visual degradation was conducted on the RIs obtained from 565 the isosurface clustering. The VQA was measured with the ${ }_{566}$ MSE-based Signal-to-Noise Ratio (SNR), the 3D SSIM [41], 567 and the proposed VQA. 


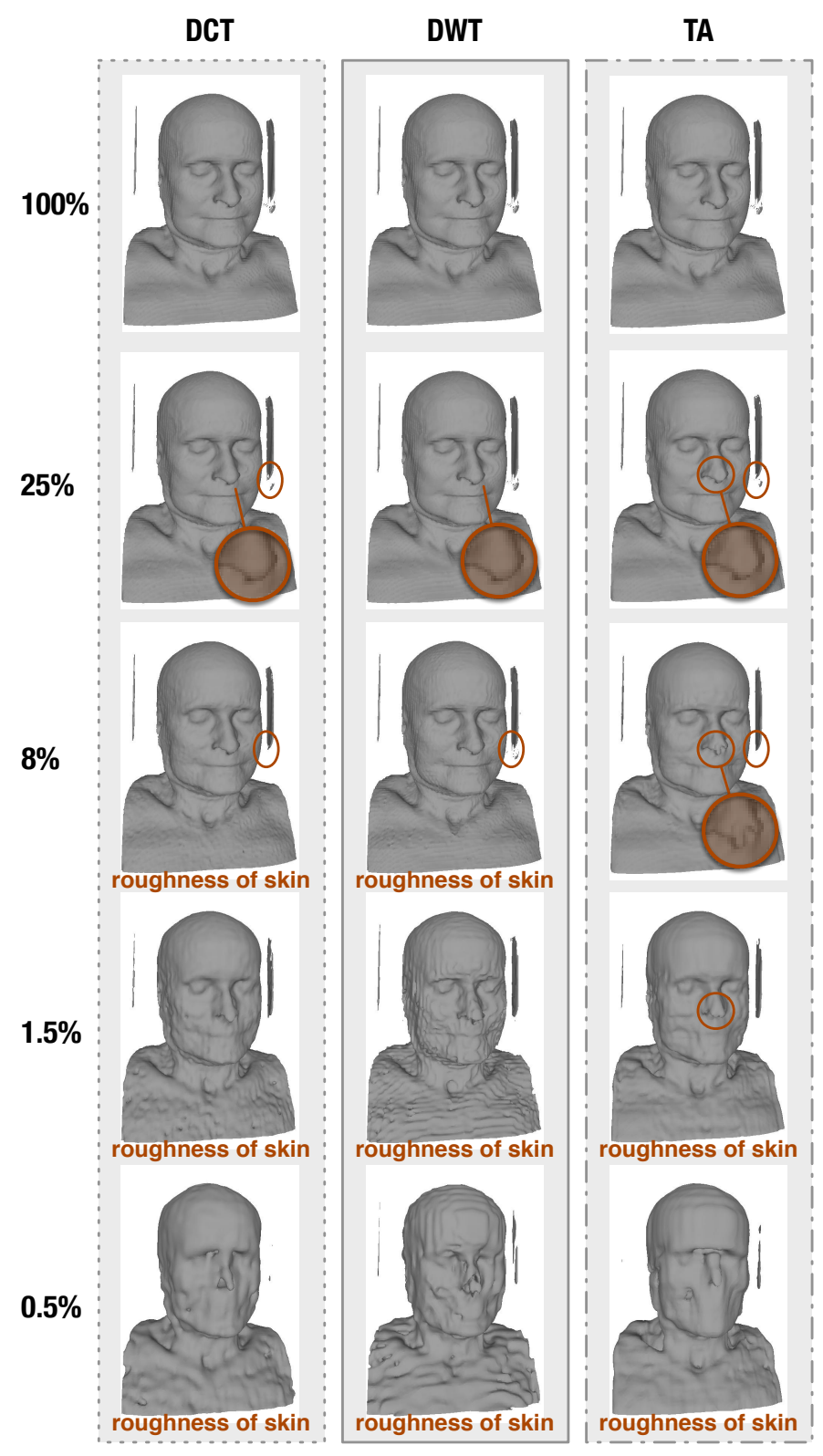

Figure 5: Visual differences of the three compression approaches on the isosurface 52 of the CT head data set. Red circles indicate significant visual differences.

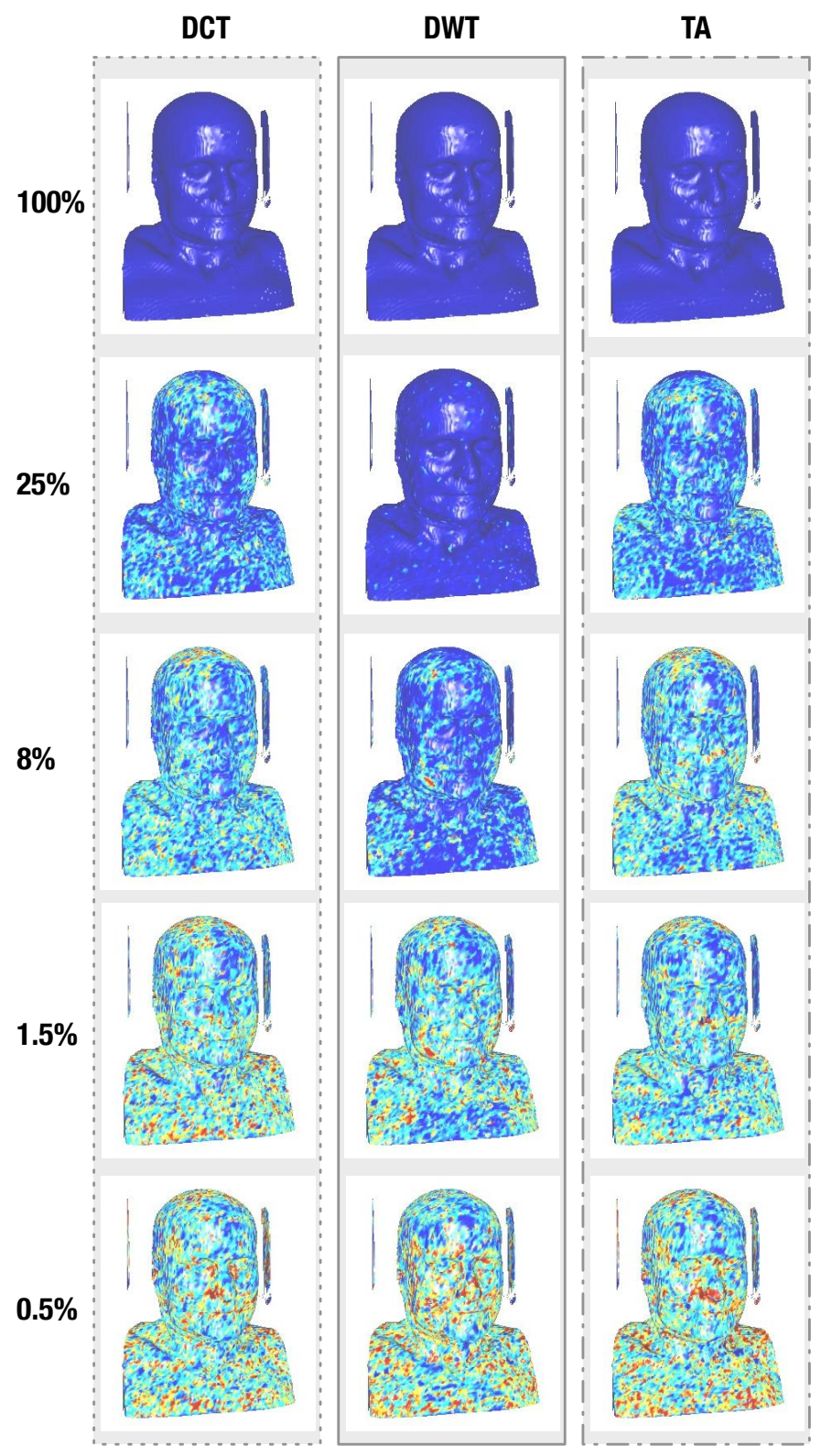

Figure 6: The distance maps of TPDM between the original isosurface 52 and the isosurfaces in Fig. 5, warmer colors represent higher local distance values, i.e., lower reconstruction quality.

\section{4.3.1. Medical data}

${ }_{569}$ As a first exemplar scalar field, we analyzed the previously 570 used CT head data set. For this purpose, we visually inspected 571 the contour degradation of the RIs from K-means on the HDM. ${ }_{572}$ Fig. 5 illustrates the visual degradation (rows) of the isosur${ }_{573}$ face $/ 3 \mathrm{D}$ contour of the skin at $\mathrm{RI}=52$ when increasing the data 574 reduction (columns) using DCT, DWT, and TA. Some notable 575 visual differences are circled in red. The first few data reduc576 tion levels show only minor differences. For example, for TA, 577 the nose starts to have a small artifact from data reduction level 578 of $25 \%$ on. The skin pattern differs quite strongly, especially 579 for compression levels of $8 \%$ and below. There, the behavior 580 of the three compression approaches is well visible. Notably, ${ }_{581}$ the DWT contours reveal an axis aligned pattern resulting from
582 the applied wavelets along the three spatial axis, while TA con583 tours show a more compact shape and a smoother skin as a re584 sult of extracting principal components of the shape. The DCT 585 contours contain a noise pattern what is also typical for a high ${ }_{586}$ DCT compression. Fig. 6 shows the distance maps produced by ${ }_{587}$ TPDM between the original contour at RI=52 and the contours 588 in Fig. 5 where warmer colors represent higher local distortions. 589 The TPDM maps are quite consistence with the visual inspec590 tion (e.g., at compression level 8\%, the nose artifact of TA is 591 clearly shown as red).

${ }_{992}$ In Fig. 7, we put the proposed VQA scores in relation to ${ }_{593}$ SNR and SSIM to analyze the degradation during increased ${ }_{594}$ data reduction levels for DCT, DWT and TA. In general, the ${ }_{595}$ curves follow a similar pattern. Interestingly, the SSIM mea- 

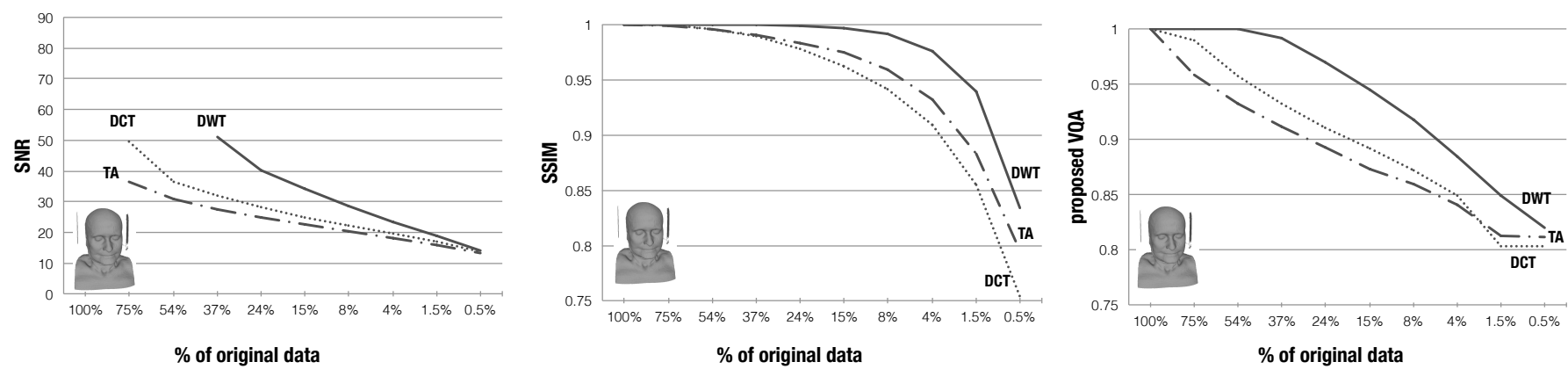

Figure 7: Comparison of the SNR, the SSIM, and the proposed VQA for assessing visual quality of volumes compressed with DCT, DWT and TA. The proposed VQA gives more insight into differences until approximately 50\% of data reduction, in particular as opposed to SSIM.

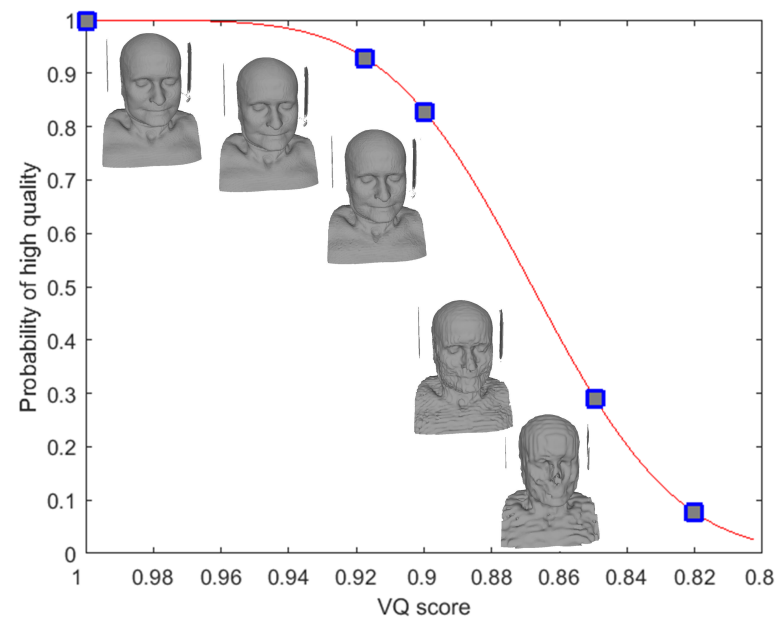

Figure 8: Psychometric function for the proposed quality metric plotted with $V Q$ score and $p$ pairs of the compressions (from left to right) at level $100 \%$, $25 \%, 8 \%, 1.5 \%$, and $0.5 \%$. The RIs at isovalue 52 serve as references for the visual degradation of different compressions. The $p$ values and the perceived degradations are linearly correlated.

596 sures a different ranking of the quality where it is DWT, TA, 597 DCT opposed to the quality ranking by SNR and our proposed ${ }_{598}$ VQA where it is DWT, DCT, TA. Referring back to Fig. 5 and ${ }_{599}$ Fig. 8, it is challenging to give a clear answer whether DCT or 600 TA should be ranked as higher visual quality. However, until a 601 data reduction level of $8 \%$, DWT clearly performs better. For 602 the data reduction levels of $1.5 \%$ and $0.5 \%$ we notice different ${ }_{603}$ degradation in the isosurface, which cannot be depicted with ${ }_{604}$ SSIM and the proposed VQA.

605 Fig. 8 plots the psychometric function where the square 606 markers (from left to right) indicate compressions at level $607100 \%, 25 \%, 8 \%, 1.5 \%$, and $0.5 \%$. We note that the probability 608 of high visual quality $p$ effectively reflects the perceived degra609 dation, where $p>0.8(V Q>0.9)$ corresponds to compressions 610 with good visual quality and the strong visual degradations at 611 the two rightmost compressions are indicated by low $p$ values. ${ }_{612}$ The worst $V Q$ score is around 0.8 which corresponds to bad 613 visual quality with $p \approx 0$.

${ }_{614}$ To evaluate the benefit of the proposed VQA, we show an ${ }_{615}$ example where the proposed VQA gives more insights than

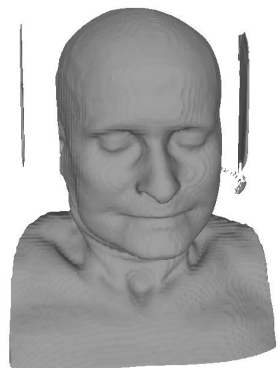

(a) Original

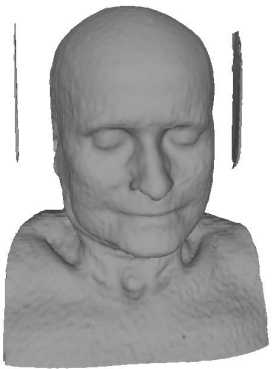

(b) DCT $8 \%$ $\mathrm{SSIM}=0.941$

$V Q_{\text {proposed }}=0.871\left(p=V Q_{\text {proposed }}=0.849(p=\right.$ $0.535) \quad 0.286)$

$V Q_{\text {equidistant }}=0.823 \quad V Q_{\text {equidistant }}=0.855$
Figure 9: Evaluation of the proposed VQA. Isosurface 52 extracted from the original volume (a), from DCT compression at data reduction level of $8 \%$ (b), and from DWT compression at data reduction level of $1.5 \%$ (c).

${ }_{616}$ SSIM. In Fig. 9 (b) and (c), we have illustrated two compressed 617 volumes, having the same SSIM scores, whose reconstructed 618 isosurfaces are of significantly different visual quality. Only ${ }_{619}$ the proposed $V Q$ scores depict the visual differences of the re620 constructed isosurfaces. The psychometric function (6) maps ${ }_{621} V Q$ scores to probabilities of high visual quality and assigns ${ }_{622} p=0.533$ to (b) and $p=0.286$ to (c). To further demonstrate 623 the effectiveness of assessing volume quality using benchmark 624 isovalues, we computed the VQA score, defined in Eq. 5, but 625 using isovalues from equidistant sampling over the isovalue

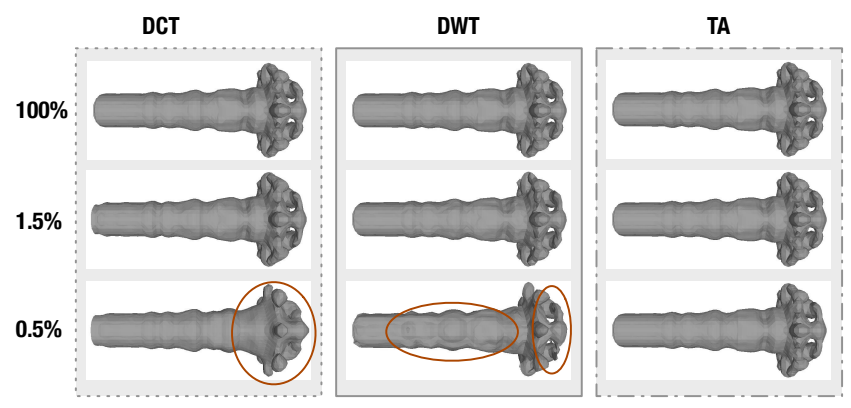

Figure 10: Visual differences of the three compression approaches on the isosurface 15 of the fuel data set. Red circles indicate significant visual differences. 

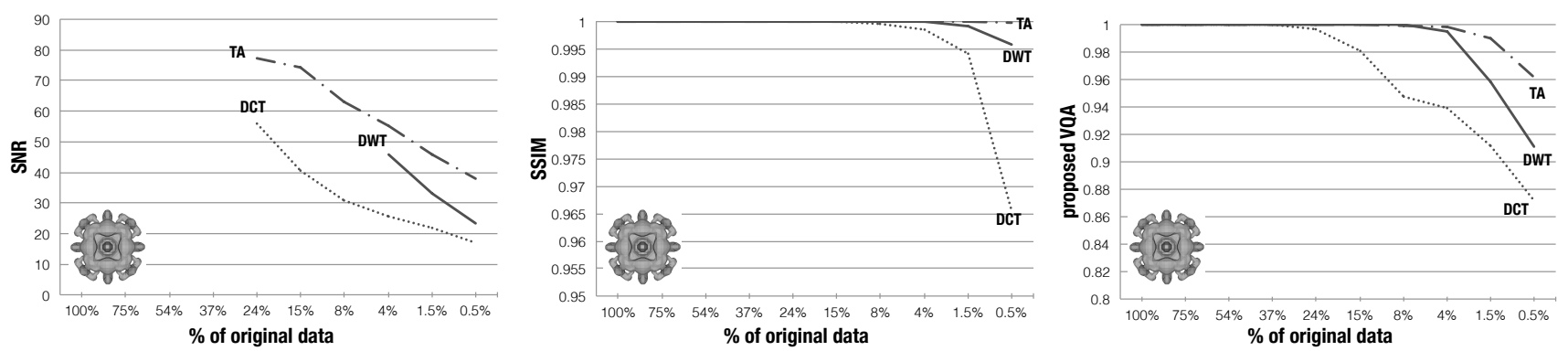

Figure 11: Comparison of the SNR, the SSIM, and the proposed VQA for assessing visual quality of volumes compressed with DCT, DWT and TA.
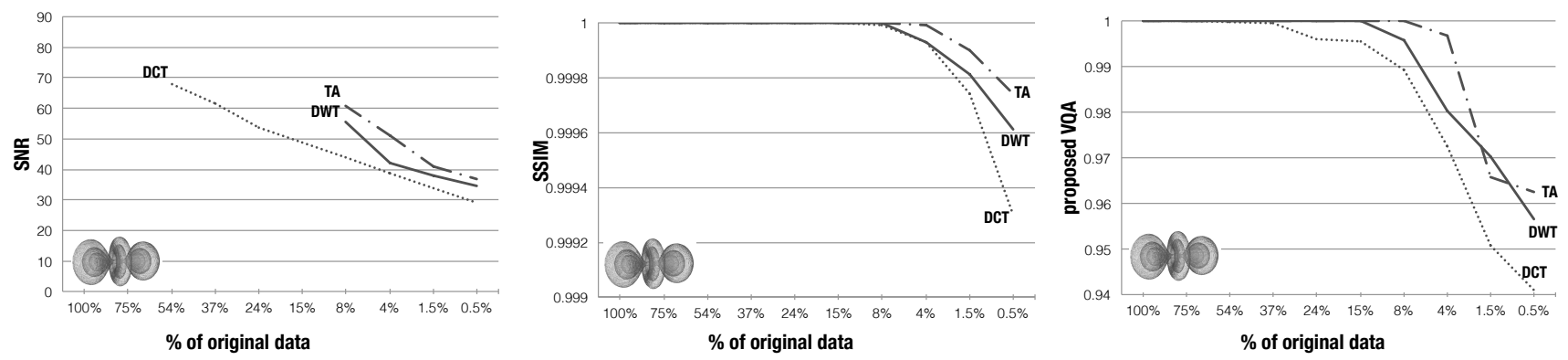

Figure 12: Comparison of the SNR, the SSIM, and the proposed VQA for assessing visual quality of volumes compressed with DCT, DWT and TA. The proposed VQA shows more differences for the medium data reduction levels.

${ }_{626}$ range. As a result, a higher score is assigned to the volume ${ }_{644}$ proaches, shown in Fig. 11, we observed again similar curve 627 in Fig. 9 (c) although it has a lower visual quality. Since the ${ }_{645}$ patterns of the three VQAs, where the SNR scores lay above 628 equidistant sampling may select unstable noisy isosurfaces in ${ }_{646} 20$ decibel and the TPDM scores are quite below the 0.2 psy629 the computation of VQA, it is less suitable to accurately assess ${ }_{647}$ chometric fitting score. All VQA curves show a perfect recon630 the volume quality.

\section{4.3.2. Simulation data}

648 struction of DWT until 8\%; TA and DCT show a perfect quality ${ }_{649}$ from $37 \%$ on for SNR and the proposed VQA. For lower per${ }_{650}$ centages, TA is consistently above DWT and DCT. In the pro${ }_{632}$ The next scalar field we analyzed is a simulation of a fuel ${ }_{651}$ posed VQA, the differences between the VQAs on 5\% are more ${ }_{633}$ injection into a combustion chamber $\left(64 \times 64 \times 64,8\right.$-bit). It ${ }_{652}$ similar than in SSIM, while all VQAs show DWT and TA to be ${ }_{634}$ basically shows the absence/presence of air with density val- ${ }_{653}$ closer to each other for $4 \%$ and $1.5 \%$.

${ }_{635}$ ues in a 3D scalar field. The degradation of isosurface 15 of ${ }_{654}$ The last 3D scalar field we analyzed with respect to VQA is ${ }_{636}$ a selected RI is depicted in Figs. 10. In general, all the com- 655 a simulation of the spatial probability electron distribution in an ${ }_{637}$ pression approaches perform quite well; nevertheless, there are 656 hydrogen atom (magnetic field) of size $128 \times 128 \times 128,8$-bit. ${ }_{638}$ some subtleties. While TA offers a nearly perfect reconstruction ${ }_{657}$ The hydrogen data sets achieves very high quality visual recon639 for all data reduction levels, especially DWT has some irregular 658 structions, which is reflected with all VQA scores, as shown ${ }_{640}$ artifacts. It is also nicely visible that DCT is receptive to peri${ }_{641}$ odic patterns what can be seen in the symmetric data reduction 642 artifacts.

659 in Fig. 12. Moreover, the differences on the extracted surfaces 660 such as in Fig. 13 for RI=26 are hardly visible. Still, the pro${ }_{661}$ posed VQA shows again more differences for the medium data

Looking at the VQA curves of the three compression ap- 662 reduction levels.

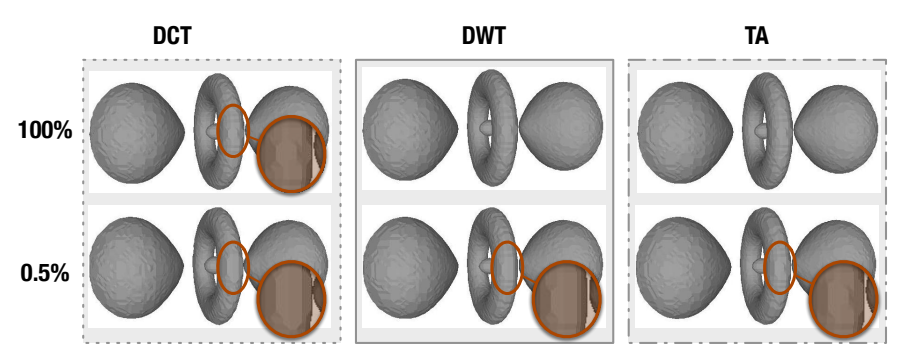

\section{4.4. VQA conclusions and future work}

664 In conclusion, we proposed a VQA based on relevant bench665 mark 3D contours to measure the visual quality degradation of ${ }_{666} 3 \mathrm{D}$ scalar fields. Even though we measure only a scarce set of 667 isosurfaces, we achieve comparable VQA scores that often even ${ }_{668}$ give more details for smaller data reduction amounts. The vi669 sual degradation in contours is a powerful information change 670 with respect to significant structures in scalar fields. While this ${ }_{671}$ work only demonstrates a possible future direction of VQA for Figure 13: Degradation of isosurface 26 extracted on scalar fields from DCT, 672 scalar fields, its details still need to be explored. For example, DWT, and TA. Red circles indicate significant visual differences. 673 varying MQA measures could be compared in the future. It also 
674 needs to be investigated further how well the vertex projection 675 steps works for isolated components in isosurfaces. Our exper676 iments indicate positive results; however, there is no guarantee 677 that this approximation works for all data sets, see, e.g., [1] page 678 17. The proposed VQA scores match well with the psychome679 tric fitting performed in [1] and the SNR values. However, for 680 low visual qualities, none of the VQAs is so far able to describe ${ }_{681}$ the pattern of the visual differences such as in Fig. 5 (bottom 682 row). Despite those limitations, we regard this demonstrated ${ }_{683}$ VQA application on RIs as an important step leading structural ${ }_{684}$ VQA for 3D scalar fields into directions already taken in related ${ }_{685}$ disciplines such as IQA and MQA.

686 Finally, the proposed comparisons by varying three compres687 sion approaches and three VQA measures show that the choice 688 of compression approach is highly application and data depen689 dent. Knowing the structure of the data and understanding how 690 much data reduction is desired, gives clues onto the choice of 691 data compression. For example, DWT looks favorable for lower 692 compression ratios as well as real data such as CT. For simula${ }_{693}$ tion data, TA is especially interesting for high data reduction 694 levels.

\section{5. Summary}

696 We have presented a novel isovalue classification algorithm 697 that extracts representative surfaces from a 3D scalar field data 698 set. Compared to previous work, we were able to detect more 699 distinct shapes with a set of isosurfaces illustrating the most 700 representative features in a 3D scalar field data set. We have 701 proposed a structural VQA based on benchmark isosurfaces to 702 assess the visual quality of the compressed 3D scalar fields. ${ }_{703}$ The VQA builds upon the proposed isovalue classification al704 gorithm. We have also demonstrated the utility of our pro705 posed VQA using three widely-used compression approaches 706 (i.e., DWT, DCT and TA).

\section{Acknowledgement}

708 This work was supported by the Swiss National Science ${ }_{709}$ Foundation (PBZHP2_147309), the US National Science Foun710 dation (NSF IIS-1617101), and the Office of Naval Research 711 (N00014-16-1-2228). The datasets are courtesy of the volvis 712 community and OsiriX Foundation.

\section{${ }_{713}$ References}

1] Torkhani F, Wang K, Chassery JM. A curvature-tensor-based perceptual quality metric for 3D triangular meshes. Machine Graphics and Vision 2014;23(1-2):59-82.

7 [2] Engel K, Hadwiger M, Kniss JM, Rezk-Salama C, Weiskopf D. Real-time Volume Graphics. AK Peters; 2006.

[3] Balsa Rodriguez M, Iglesias Guitián JA, Gobbetti E, Makhinya M, Marton F, Pajarola R, et al. State-of-the-art in compressed GPU-based direct volume rendering. Computer Graphics Forum 2014;33(6):77-100. doi:10.1111/cgf.12280.

[4] Lindstrom P. Fixed-rate compressed floating-point arrays. IEEE Transactions on Visualization and Computer Graphics 2014;20(12):2674-83. doi:10.1109/TVCG.2014.2346458.
[5] Lindstrom P, Chen P, Lee EJ. Reducing disk storage of full-3D seismic waveform tomography (F3DT) through lossy online compression. Computers \& Geosciences 2016;93:45 - 54. doi:http://dx.doi.org/10.1016/j.cageo.2016.04.009.

[6] Wang Z, Bovik AC. Mean squared error: Love it or leave it? A new look at signal fidelity measures. IEEE Signal Processing Magazine 2009;26(1):98-117. doi:10.1109/MSP.2008.930649.

[7] Corsini M, Larabi MC, Lavoué G, Petřík O, Váša L, Wang K. Perceptual Metrics for Static and Dynamic Triangle Meshes. Computer Graphics Forum 2013;32(1):101-25. doi:10.1111/cgf.12001.

[8] Suter SK, Zollikofer CP, Pajarola R. Application of tensor approximation to multiscale volume feature representations. In: Proceedings Vision, Modeling, and Visualization Workshop. 2010, p. 203-10.

[9] Hansen CD, Chen M, Johnson CR, Kaufman AE, Hagen H. Scientific Visualization: Uncertainty, Multifield, Biomedical, and Scalable Visualization. Springer; 2014.

[10] Ljung P, Lundstrom C, Ynnerman A, Museth K. Transfer function based adaptive decompression for volume rendering of large medical data sets. In: Proceedings of the 2004 IEEE Symposium on Volume Visualization and Graphics. 2004, p. 25-32. doi:10.1109/VV.2004.14.

[11] Bruckner S, Möller T. Isosurface similarity maps. Computer Graphics Forum 2010;29(3):773-82. doi:10.1111/j.1467-8659.2009.01689.x.

[12] Jones M, Baerentzen J, Sramek M. 3D distance fields: A survey of techniques and applications. IEEE Transactions on Visualization and Computer Graphics 2006;12(4):581-99. doi:10.1109/TVCG.2006.56.

[13] Chiueh TC, Yang CK, He T, Pfister H, Kaufman AE. Integrated volume compression and visualization. In: Proceedings IEEE Visualization. Computer Society Press; 1997, p. 329-36. doi:10.1109/VISUAL.1997.663900.

[14] Yeo BL, Liu B. Volume rendering of DCT-based compressed 3D scalar data. IEEE Transactions on Visualization and Computer Graphics 1995;1(1):29-43. doi:10.1109/2945.468390.

[15] Meftah A, Antonini M. Scan-based wavelet transform for huge 3D volume data. In: Proceedings Picture Coding Symposium. 2009, p. 1-4. doi:10.1109/PCS.2009.5167423.

[16] Parys R, Knittel G. Giga-voxel rendering from compressed data on a display wall. In: Proceedings International Conference in Central Europe on Computer Graphics, Visualization and Computer Vision. 2009, p. 1522.

[17] Gobbetti E, Iglesias Guitián J, Marton F. COVRA: A compressiondomain output-sensitive volume rendering architecture based on a sparse representation of voxel blocks. Computer Graphics Forum 2012;31:1315-24. doi:10.1111/j.1467-8659.2012.03124.x.

[18] Suter SK, Makhinya M, Pajarola R. TAMRESH: Tensor approximation multiresolution hierarchy for interactive volume visualization. Computer Graphics Forum 2013;32(8):151-60. doi:10.1111/cgf.12102.

[19] Wang Z, Bovik AC, Sheikh HR, Simoncelli EP. Image quality assessment: from error visibility to structural similarity. IEEE Transactions on Image Processing 2004;13(4):600-12.

[20] Lin W, Kuo CCJ. Perceptual visual quality metrics: A survey. Journal of Visual Communication and Image Representation 2011;22(4):297-312. doi:10.1016/j.jvcir.2011.01.005.

[21] Zhang L, Zhang L, Mou X, Zhang D. FSIM: A feature similarity index for image quality assessment. IEEE Transactions on Image Processing 2011;20(8):2378-86. doi:10.1109/TIP.2011.2109730.

[22] Pinto GO, Rouse DM, Hemami SS. Measuring contour degradation in natural image utility assessment: Methods and analysis. In: Proceedings SPIE; vol. 7865. 2011, p. 78650U-. doi:10.1117/12.877102.

[23] Liu A, Liu W, Narwaria M. Image quality assessment based on gradient similarity. IEEE Transactions on Image Processing 2012;21(4):1500-12. doi:10.1109/TIP.2011.2175935.

[24] Zhu J, Wang N. Image quality assessment by visual gradient similarity. IEEE Transactions on Image Processing 2012;21(3):919-33. doi:10.1109/TIP.2011.2169971.

[25] Janicke H, Chen M. A salience-based quality metric for visualization. Computer Graphics Forum 2010;29(3):1183-92. doi:10.1111/j.14678659.2009.01667.x.

3 [26] Athawale T, Entezari A. Uncertainty quantification in linear interpolation for isosurface extraction. IEEE Transactions on Visualization and Computer Graphics 2013;19(12):2723-32. doi:10.1109/TVCG.2013.208.

[27] Athawale T, Sakhaee E, Entezari A. Isosurface visualization of data with 

tion and Computer Graphics 2016;22(1):777-86.

99 [28] Sakhaee E, Entezari A. A statistical direct volume rendering framework for visualization of uncertain data. IEEE Transactions on Visualization and Computer Graphics 2016 (in press); doi:10.1109/TVCG.2016.2637333.

[29] Lorensen WE, Cline HE. Marching Cubes: A high resolution 3D surface construction algorithm. ACM SIGGRAPH Computer Graphics 1987;21(4):163-9.

60 [30] Cignoni P, Rocchini C, Scopigno R. Metro: Measuring error on simplified surfaces. In: Computer Graphics Forum; vol. 17. 1998, p. 167-74.

808 [31] Bronstein AM, Bronstein MM. Handbook of Mathematical Methods in Imaging; chap. Manifold Intrinsic Similarity. Springer; 2011, p. 1403-52.

810 [32] Du Q, Faber V, Gunzburger M. Centroidal voronoi tessellations: applications and algorithms. SIAM review 1999;41(4):637-76. doi:10.1137/S0036144599352836.

333] Liu Y, Wang W, Lévy B, Sun F, Yan DM, Lu L, et al. On centroidal voronoi tessellation - energy smoothness and fast computation. ACM Transactions on Graphics 2009;28(4):101:01-17. doi:10.1145/1559755.1559758.

[34] Suter SK, Ma B, Entezari A. Visual analysis of 3D data by isovalue clustering. In: Proceedings International Symposium on Visual Computing. 2014, p. 313-22. doi:10.1007/978-3-319-14249-4_30.

[35] Pelleg D, Moore AW. X-means: Extending K-means with efficient estimation of the number of clusters. In: Proceedings International Conference on Machine Learning. 2000, p. 727-34.

[36] Shahbaba M, Beheshti S. Improving X-means clustering with MNDL. In: Proceedings International Conference on Information Science, Signal Processing and their Applications. 2012, p. 1298-302. doi:10.1109/ISSPA.2012.6310493.

[37] Kulis B, Jordan MI. Revisiting k-means: New algorithms via Bayesian nonparametrics. In: Proceedings International Conference on Machine Learning. 2012, p. 513-20.

[38] Lavoué G, Drelie Gelasca E, Dupont F, Baskurt A, Ebrahimi T. Perceptually driven $3 \mathrm{D}$ distance metrics with application to watermarking. In: SPIE Applications of Digital Image Processing XXIX. 2006, p. 63120L.

[39] Lavoué G. A multiscale metric for 3D mesh visual quality assessment 2011;30(5):1427-37.

35 [40] Ballester-Ripoll R, Suter SK, Pajarola R. Analysis of tensor approximation for compression-domain volume visualization. Computers \& Graphics 2015;47:34-47. doi:10.1016/j.cag.2014.10.002.

38 [41] Zeng K, Wang Z. 3D-SSIM for video quality assessment. In: 839 IEEE International Conference on Image Processing. 2012, p. 621-4. 840 doi:10.1109/ICIP.2012.6466936. 
